\title{
Comunicación
}

\section{Uso de un simulador bovino para prácticas de palpación transrectal}

\author{
Use of a bovine simulator for transrectal palpation practices
}

\author{
Andrea Baracaldo-Martinez ${ }^{1}$, Pablo Domínguez-Castaño ${ }^{1}$, Erika Natalia Franco- \\ Hernández ${ }^{1}$, Jorge Eduardo Atuesta-Bustos ${ }^{1}$, Diego Alejandro Robayo-Triviño ${ }^{2,3}$
}

\section{Resumen}

El objetivo del estudio fue determinar la eficiencia de un simulador bovino para prácticas de palpación transrectal. Se utilizó un simulador bovino comercial y cuatro hembras bovinas no gestantes. Se trabajó con 20 y 22 estudiantes sin experiencia en la técnica de palpación transrectal que participaron como grupo experimental (I) y control (II), respectivamente. Los estudiantes del grupo I fueron entrenados con el simulador para la ubicación de cérvix, útero y ovarios, mientras que los del grupo II recibieron una práctica tradicional, consistente en el trabajo con tractos reproductivos in situ. Luego, ambos grupos fueron evaluados por dos palpadores expertos en cuatro bovinos respecto a la correcta ubicación de las estructuras anatómicas. La prueba de Chi cuadrado determinó diferencias en el grado de precisión del reconocimiento de las estructuras anatómicas entre grupos $(\mathrm{p}<0.05)$, habiendo un mejor nivel de reconocimiento de las estructuras en el grupo experimental, indicando la utilidad del simulador para la identificación de la cérvix, útero y ovarios en las prácticas de palpación transrectal.

Palabras clave: bienestar animal; bovinos; palpación; reproducción

${ }^{1}$ Facultad de Ciencias Agrarias, Fundación Universitaria Agraria de Colombia, Bogotá, Colombia

${ }^{2}$ Escuela de Ciencias Agrícolas, Pecuarias y del Medio Ambiente, Universidad Nacional Abierta y a Distancia, Bogotá, Colombia

${ }^{3}$ E-mail: diego.robayo@unad.edu.co

Recibido: 30 de noviembre de 2018

Aceptado para publicación: 28 de junio de 2019 
The aim of this study was to determine the efficiency of a bovine simulator for transrectal palpation practices. A commercial bovine simulator and four non-pregnant bovine females were used. The study included 20 and 22 students without experience in transrectal palpation who participated in the experimental (I) and control (II) group, respectively. The students of group I were trained with the simulator for the location of cervix, uterus and ovaries, while those of group II received a traditional practice, consisting of working with reproductive tracts in situ. Then, both groups were evaluated by two expert instructors in four cows regarding the correct location of the anatomical structures. The Chi square test determined differences in the degree of accuracy of the recognition of the anatomical structures between groups $(p<0.05)$, having a better level of recognition of the structures in the experimental group, which indicated the utility of the simulator for the identification of the cervix, uterus and ovaries in transrectal palpation practices.

Key words: animal welfare; cattle; palpation; reproduction

\section{INTRODUCCIÓN}

La Organización Mundial de Sanidad Animal (OIE) ha identificado el bienestar animal como una de las prioridades de su tercer Plan Estratégico (Huertas et al., 2014). Esto ha determinado que la implementación de los conceptos de bienestar animal en el uso de animales vivos para la realización de prácticas en cursos relacionados con la producción animal sea más complejo, dado que muchas de estas actividades, entre ellas las relacionadas a la reproducción animal, requieran de procesos de tipo invasivo y de carácter repetitivo, como lo son las palpaciones transrectales y la manipulación del cérvix, entre otros, los cuales pueden generar estrés en los animales, y en ocasiones pueden llegar a afectar la salud y la productividad animal (Steele, 2013; Jaskowski et al., 2019).

La utilización de simuladores en la enseñanza universitaria permite un adiestramiento práctico en situaciones similares a las reales para que el estudiante se capacite en la técnica, a través de repetir múltiples veces una maniobra que le permita asegurar su correcta realización antes de aplicarlo en individuos reales (Deakin et al., 2010). En el campo de la Medicina Veterinaria se han venido utilizando bio-simuladores y maniquíes de fibra de vidrio en los que se introducen órganos de animales ex-vivo en técnicas de cirugía laparoscópica para realizar procedimientos básicos de aprendizaje (Molina et al., 2012).

En este mismo sentido, se reconoce que el uso estructurado de este tipo de equipos en la enseñanza-aprendizaje puede ser integrado en los currículos de los cursos como una herramienta de entrenamiento y evaluación de las competencias adquiridas por los estudiantes (Dávila-Cervantes, 2014). No obstante, el número de reportes que evalúen la eficiencia de estos simuladores son escasos. Es así que el objetivo de este estudio fue evaluar la eficiencia de un simulador bovino para prácticas de palpación transrectal que permita su inclusión en las prácticas de reproducción animal de estudiantes de programas relacionados con las ciencias animales.

\section{Materiales Y Métodos}

El trabajo con el simulador bovino se llevó a cabo en las instalaciones de la Universidad Nacional Abierta y a Distancia, Bogotá, 
Colombia y la posterior comparación con animales in situ se llevó a cabo en el Centro de Investigación y Desarrollo Tecnológico (CIDT) de la Fundación Universitaria Agraria de Colombia (UNIAGRARIA), ubicada en La Punta, Cundinamarca. Esta zona se encuentra a una altitud de $2587 \mathrm{msnm}$, con temperatura promedio de $13{ }^{\circ} \mathrm{C}$ y humedad relativa de $70 \%$.

Se utilizó el Simulador Bovino F1 de la empresa Embriovinos S.A.S. (Colombia), el cual es una representación a escala en tamaño natural de una hembra bovina. El simulador es fabricado en fibra de vidrio, resinas, catalizador, látex, dos pinturas plásticas impermeabilizantes ( 7 y p4), pinturas acrílicas, espuma con memoria, poliuretano y rubatex. Paras las prácticas de palpación transrectal el simulasdor cuenta con un tracto reproductor que simula cérvix, cuerpo y cuernos uterinos, y dispone de 12 ovarios con diferentes estructuras foliculares y luteales, así como conductos vaginales de látex.

Para la identificación de estructuras reproductivas in vivo se utilizaron cuatro hembras bovinas Holstein, no gestantes, con edades entre 4 y 6 años, que eran rutinariamente utilizadas para prácticas de reproducción en el CIDT.

Se trabajó con 20 estudiantes de tercer año de la carrera de Zootecnia, escogidos de una lista de voluntarios, donde el criterio de inclusión fue que los estudiantes no tuvieran un entrenamiento previo en la técnica de palpación transrectal (grupo experimental). Los estudiantes fueron subdivididos aleatoriamente en cuatro grupos y recibieron una capacitación previa de dos horas en anatomía reproductiva y fisiología del ciclo estral, después recibieron entrenamiento con un único simulador bovino para la identificación de las estructuras anátomicas cérvix, útero (cuerpo y cuernos) y ovarios durante un periodo de cuatro horas.
Posteriormente, en grupos de máximo cinco estudiantes trabajaron con los animales in vivo para la identificación de las tres estructuras anatómicas indicadas. Cada estudiante participante palpó a las cuatro vacas experimentales obteniendo un total de cuatro repeticiones por cada estudiante. La correcta ubicación anatómica expresada por el estudiante fue corroborada por dos docentes expertos en la técnica de palpación transrectal, evaluando cada uno a la mitad del grupo. La corroboración de la correcta ubicación de la estructura anatómica consistió en que cada estudiante expresaba estar palpando una estructura anatómica determinada (cérvix, útero u ovarios) y esto era corroborado por el docente introduciendo la mano a través del recto del animal. El periodo de descanso que tuvieron las vacas entre grupos de palpación fue de una semana.

En forma paralela, se trabajó con 22 estudiantes de tercer año del programa de Zootecnia, escogidos de una lista de voluntarios (grupo control), los cuales recibieron una capacitación similar al grupo experimental sobre anatomía reproductiva y fisiología del ciclo estral. El criterio de inclusión fue el mismo que para el grupo experimental es decir que los estudiantes no tuvieran un entrenamiento previo en la técnica de palpación transrectal. Estos estudiantes fueron distribuidos en cinco grupos, los cuales realizaron la práctica tradicional en palpación transrectal, que consistió en trabajar con cinco tractos reproductores bovinos obtenidos del centro de beneficio local para el reconocimiento de las estructuras (cérvix, útero y ovarios), y posteriormente, trabajar con los animales in vivo para la identificación de estas estructuras, siguiendo el procedimiento indicado para los estudiantes del grupo experimental.

Las diferencias estadísticas en el grado de precisión del reconocimiento de las estructuras anatómicas entre los dos grupos de estudiantes fueron determinadas mediante la prueba de Chi-cuadrado utilizando el software estadístico STATA ${ }^{\circledR}$ 
Cuadro 1. Frecuencia de reconocimiento positivo de estructuras reproductivas mediante palpación rectal en cuatro vacas por estudiantes de Medicina Veterinaria

\begin{tabular}{lcccccc}
\hline \multirow{2}{*}{ Grupo } & \multicolumn{6}{c}{ Estructuras } \\
\cline { 2 - 7 } & \multicolumn{2}{c}{ Cérvix } & \multicolumn{2}{c}{ Útero } & \multicolumn{2}{c}{ Ovarios } \\
\hline Experimental $(\mathrm{n}=20)$ & 56 & $70.0^{\mathrm{a}}$ & 20 & $25.0^{\mathrm{a}}$ & 15 & $18.8^{\mathrm{a}}$ \\
Control $(\mathrm{n}=23)$ & 49 & $53.3^{\mathrm{b}}$ & 7 & $7.6^{\mathrm{b}}$ & 7 & $7.6^{\mathrm{b}}$ \\
\hline a,b Letras diferentes dentro de columnas indican diferencias estadísticas significativas $(\mathrm{p}<0.05)$
\end{tabular}

\section{Resultados y Discusión}

Los estudiantes del grupo experimental presentaron un mayor grado de precisión para la identificación del cérvix, el útero y los ovarios, en comparación con los estudiantes del grupo control $(\mathrm{p}<0.05)$ (Cuadro 1).

Los resultados obtenidos en este estudio son similares a los presentados por Baillie et al. (2005a), quienes trabajaron con estudiantes de medicina veterinaria de tercer año sin entrenamiento previo en palpación transrectal y con un simulador bovino háptico en fibra de vidrio PHANToM $\AA$, encontrando diferencias significativas en el porcentaje de la correcta ubicación anatómica del útero in vivo entre estudiantes que practicaron con el simulador (56.2\%) y estudiantes que no lo hicieron (3.1\%). Este mismo grupo obtuvo respuestas similares en un estudio posterior trabajando con el simulador bovino háptico PHANToM premium 1.5® (Baillie et al., 2010).

Es posible que los mejores resultados obtenidos por los estudiantes que practican con el simulador puedan deberse a una mayor autoconfianza para desarrollar el proceso de palpación transrectal con animales in vivo, ya que es una práctica adicional y reciben orientación directa por parte del profesor acerca de los fallos que podrían estar teniendo al desarrollar la técnica de palpación transrectal (Baillie et al., 2005b). Por otro lado, el escenario de un simulador representa un ambiente seguro donde no se presentan consecuencias negativas por los errores que puedan presentar los aprendices y donde pueden aprender y corregir rápidamente los posibles errores (Zolhavarieh et al., 2016).

En contraste con los resultados obtenidos, Bossaert et al., 2009, reportan mejores resultados de acierto de la ubicación anatómica del útero y de los ovarios en vacas vivas entre estudiantes que practicaban directamente con animales en planta de beneficio $(60 \%)$ comparados con estudiantes que practicaban con el simulador bovino Breed'n Betsy ${ }^{\circledR}(28 \%)$, planteando que el simulador falló en proveer condiciones reales como la presión y movimientos peristálticos del recto.

Es importante tener en cuenta, que el uso de simuladores como herramienta para la educación en programas de Medicina Veterinaria y Ciencias Animales es de gran importancia debido a que en las prácticas con animales se tiene un mayor riesgo de realizar un mal procedimiento, debido a la falta de destreza e inseguridad para la toma de decisiones que puedan tener los estudiantes (Cruz, 2016).

\section{Literatura Citada}

1. Baillie S, Crossan A, Brewter SA, Stephen A, May A, Mellor D, Reid S. 2005. Validation of a bovine rectal palpation simulator for training veterinary students. Stud Health Technol Inform 111: 33-36. 
2. Baillie S, Mellor D, Brewster SA, Reid $S W .2005 a$. Integrating a bovine rectal palpation simulator into an undergraduate veterinary curriculum. J Vet Med Educ 32: 79-85.

3. Baillie $S$, Crossan A, Brewter SA, May SA, Mellor DJ. 2010. Evaluating an automated haptic simulator designed for veterinary students to learn bovine rectal palpation. Simul Healthc 5: 261266. doi: 0.1097/SIH.0b013e3181e369bf

4. Bossaert P, Leterme L, Caluwaerts T, Cools S, Hostens M, Kolkman I de Kruif A. 2009. Teaching transrectal palpation of the internal genital organs in cattle. J Vet Med Educ 36: 451-460. doi: $10.3138 /$ jvme. 36.4 .451

5. Cruz S. 2016. El uso de simuladores como herramienta de aprendizaje en la enseñanza de medicina veterinaria. Tesis de Especialización. Bogotá: Univ. Militar Nueva Granada. 27 p.

6. Dávila-Cervantes A. 2014. Simulación en educación médica. Inv Ed Med 3: 100-105. doi: 10.1016/S2007-5057(14)72733-4

7. Deakin CD, Nolan JP, Soar J, Sunde K, Koster RW, Smith GB, Perkins GD. 2010. European resuscitation council guidelines for resuscitation 2010 section 4. Adult advanced life support. Resuscitation 81: 1305-1352. doi: 10.1016/ j.resuscitation.2010.08.017
8. Huertas S, Gallo C, Galindo F. 2014. Motores de las políticas de bienestar animal en las Américas. Rev Sci Tech OIE 33: 55-66.

9. Jaskowski J, Kaczmarowski M, Kulus J, Jaskowski B, Herudzinska M, Gehrke. 2019. Rectal palpation for pregnancy in cows: A relic or an alternative to modern diagnostic methods. Med Weter 75: 259-264. doi: 10.21521/mw.6156

10. Molina J, Silveira E, Heredia D, Fernández D, Bécquer L, Gómez, T, González Y, Castro M. 2012. Los simuladores y modelos experimentales en el desarrollo de habilidades quirúrgicas en el proceso de enseñanza-aprendizaje de las ciencias de la salud. REDVET 13(6).

11. Steele G. 2013. The effect of rectal palpation on milk production, cortisol level and behavior in dairy cattle. Cattle Pract 21: 14-20.

12. Zolhavarieh SM, Sadeghi-Nasab A, Ghanbari S, Mirshokraei P, Ruhi Athar M. 2016. Preliminary evaluation of learning performance on the simplest bovine trans-rectal palpation phantom for training veterinary students. Iranian $\mathrm{J}$ Ruminants Health Res 1: 21-30. doi: 10.22055/ijrhr.2016.12319 